\title{
A LUNI-SOLAR PRECESSION AND NUTATION ANALYSIS FROM RADIO ASTROMETRIC OBSERVATIONS
}
A.H.Andrei ${ }^{1}$
B.Elsmore ${ }^{2}$

1. CNPq Observatorio Nacional, Rio de Janeiro, Brasil

2. Mullard Radio Astronomy Observatory, Cambridge, UK

The Cambridge 5-km Radio Interferometer astrometric results from 1972 to 1987 were analysed, leading to corrections to the main terms of luni-solar precession and nutation. The method used follows Elsmore's 1976 suggestion (MNRAS 177, 291) which takes pairs of sources nearly 12 -h apart in right ascension.

Using sources whose positions would maximize the effects, 773 observations, all at $5 \mathrm{GHz}$, of 21 extragalactic radio sources were considered. No selection criterion was used other than that of a minimum standard of quality of the observations. The results were grouped per source, per season, forming a total of 110 groups.

The main result shows the following corrections to the 1964 IAU System. Constant of luni-solar precession, centennial: $\Delta p_{1}=1^{\prime \prime} .11 \pm 0^{\prime \prime} .15,18.6 \mathrm{y}$ term of nutation in longitude $\Delta N=0^{\prime \prime} .05 \pm 0^{\prime \prime} .01$. Details are in preparation for publication in Monthly Notices of the Royal Astronomical Society. 